\title{
Output-Only Modal Analysis Using Continuous-Scan Laser Doppler Vibrometry and Application to a $20 \mathrm{~kW}$ Wind Turbine
}

\author{
Shifei Yang \\ Graduate Research Assistant, Ph.D. Candidate \\ syang66@wisc.edu \\ $\&$ \\ Matthew S. Allen, \\ Assistant Professor \\ msallen@engr.wisc.edu \\ Department of Engineering Physics \\ University of Wisconsin-Madison \\ 535 Engineering Research Building \\ 1500 Engineering Drive \\ Madison, WI 53706
}

\begin{abstract}
:
Continuous-scan laser Doppler vibrometry (CSLDV) is a method whereby one continuously sweeps the laser measurement point over a structure while measuring, in contrast to the conventional scanning LDV approach where the laser spot remains stationary while the response is collected at each point. The continuousscan approach can greatly accelerate measurements, allowing one to capture spatially detailed mode shapes along a scan path in the same amount of time that is typically required to measure the response at a single point. The method is especially beneficial when testing large structures, such as wind turbines, whose natural frequencies are very low and hence require very long time records. Several CSLDV methods have been presented that employ harmonic excitation or impulse excitation, but no prior work has performed CSLDV with an unmeasured, broadband random input. This work extends CSLDV to that class of input, developing an outputonly CSLDV method (OMA-CSLDV). This is accomplished by adapting a recently developed algorithm for linear time-periodic systems to the CSLDV measurements, which makes use of harmonic power spectra and the harmonic transfer function concept developed by Wereley. The proposed method is validated on a randomly excited free-free beam, where one-dimensional mode shapes are captured by scanning the laser along the length of the beam. The natural frequencies and mode shapes are extracted from the harmonic power spectrum of the vibrometer signal and show good agreement with the first seven analytically-derived modes of the beam. The method is then applied to identify the shapes of several modes of a $20 \mathrm{~kW}$ wind turbine using a ground based laser and with only a light breeze providing excitation.
\end{abstract}

\section{Introduction}

Continuous-Scan Laser Doppler Vibrometry (CSLDV) is a novel method of employing a laser vibrometer in which the laser spot sweeps over the structure continuously while measuring, capturing the response of the structure from a moving measurement point. Various methods have been devised to determine the mode shapes of the structure everywhere along the scan path from as few as one such measurement. Hence, the method can greatly accelerate measurements from structures with low frequency modes, which require long time records at each measurement point when the conventional point-by-point scanning method is used. Indeed, CSLDV may be the only viable method for obtaining mode shape measurements from structures that change with time or which are subjected to inputs that are difficult to replicate. One may also obtain measurements with greatly increased spatial detail, leading to insights into the dynamics of a structure that may be helpful when performing model correlation and updating [1].

Although Sriram et al. were the first to publish regarding CSLDV in early 1990's [2-4], the research group at Imperial College in London (Stanbridge, Martarelli, Ewins and Di Maio) had been working with moving sensors 
some time previously and are responsible for most of the advancements in this area.. Stanbridge, Martarelli and Ewins coined the name "CSLDV" and developed a number of algorithms that extract one and two-dimensional operating deflection shapes from CSLDV measurements under sinusoidal and impact excitation [5-9]. Other notable contributions include the work by Vanlanduit et al. [10], who presented a CSLDV method that uses multisine excitation (periodic broadband excitation). Allen \& Sracic explored the use of higher scan frequencies together with impact excitation, presenting the lifting technique that allows conventional modal analysis curve fitting methods and tools such as the CMIF to be applied to CSLDV measurements [11-13]. They also presented a method for mass-normalizing the mode vectors obtained by CSLDV when the input force has been measured $[13,14]$. All of the existing CSLDV methods are valid only for the case where the input is either zero (free response) or else it follows a specific form and is carefully controlled. In practice one cannot always apply an input of one of these forms to a structure, but unmeasured random input forces may be present. For example, wind turbines are difficult to adequately excite due to their large size and mass, yet each day the wind provides a convenient, broadband source of ambient excitation.

This work extends CSLDV to Output-only Modal Analysis (OMA), identifying modes from responses under an unmeasured, broadband random input. Since the mode shapes of a linear system are functions of position, the CSLDV measurement appears to be from a time-periodic system when the laser spot moves in a periodic, closed scan path. A few system identification strategies have been proposed for linear time-periodic systems, as discussed in [11]. This work utilizes a method recently presented by Allen et al. in $[15,16]$ that is based on the spectra of the output of a linear time periodic system when it is excited by a broadband random input. The method utilizes the harmonic transfer function concept by Wereley and Hall, which is an extension to the concept of a frequency response function (FRF) to linear time-periodic systems [17-19]. This method can be thought of as an extension of Output-only Modal Analysis [20-25] or the Natural Excitation Technique (NExT) [26] to timeperiodic systems.

The rest of this paper is organized as follows. Section 2 reviews the proposed output-only identification technique with particular emphasis on how to interpret the response spectra of an LTP system. In Section 3 the proposed technique is demonstrated by identifying the mode shapes of a free-free beam under random excitation, and the performance of the method and effect of the chosen scan frequency are discussed. The method is then applied to a CSLDV measurement of a wind turbine blade in Section 4, and Section 5 summarizes the conclusions.

\section{Theoretical Basis}

A Linear Time Invariant (LTI) structure can be modeled with the following well known equation of motion,

$$
M \ddot{y}+C \dot{y}+K y=F(t)
$$

which can be written in an equivalent state space form as follows with $x=\left[y^{\mathrm{T}}, \dot{y}^{\mathrm{T}}\right]^{\mathrm{T}}$

$$
\begin{aligned}
& \dot{x}=A x+B u \\
& y=C x+D u
\end{aligned}
$$

When a laser vibrometer is used so that the velocity is measured, $D=0$. One can diagonalize the modal equations for the system using $x=P q$ where,

$$
P=\left[\begin{array}{c}
\Psi \\
\Psi \Lambda
\end{array}\right]
$$

with $\Psi$ a matrix of the complex modes of the system $\Psi=\left[\begin{array}{lll}\psi_{1} & \cdots & \psi_{N}\end{array}\right]$ and $\Lambda$ a diagonal matrix containing the eigenvalues of the system $\Lambda=\operatorname{diag}\left(\begin{array}{lll}\lambda_{1} & \cdots & \lambda_{N}\end{array}\right)$, with $\lambda_{r}=-\zeta_{r} \omega_{r}+j \omega_{r} \sqrt{1-\zeta_{r}^{2}}$ in terms of the $r$ th natural frequency $\omega_{r}$ and damping ratio $\zeta_{r}$ of the system. The equations of motion become

$$
\begin{aligned}
& \dot{q}=\Lambda q+P^{-1} B u \\
& y=C P q
\end{aligned}
$$


The output matrix $\mathrm{C}$ can be taken to be a matrix with all zeros and a one at the location of the nodal point that the laser is measuring. If the $k$ th measurement point is being measured, then $C P$ is a $1 \mathrm{xN}$ matrix $C P=\left[\begin{array}{lll}\psi_{k 1} & \cdots & \psi_{k N}\end{array}\right] \Lambda$. On the other hand, when the continuous-scan approach is employed with a periodic scan pattern, the laser position changes periodically with time so $C P$ becomes a periodic $1 \mathrm{xN}$ matrix of mode shapes that seem to change with time since the measurement point is moving. This can be written as $C(t) P=\left[\begin{array}{lll}\psi_{1}(t) & \cdots & \psi_{N}(t)\end{array}\right] \Lambda$ with the condition that $\psi_{r}\left(t+T_{A}\right)=\psi_{r}(t)$, and where it is understood that the mode shapes are written as time periodic functions only because the measurement point is changing with time. Hence, a system under CSLDV measurement can be modeled with a set of linear time periodic (LTP) equations of motion. The CSLDV system has $A, B$ and $D$ constant and $C$ time varying. In the most general case, the response of a linear time periodic system can be written as follows in terms of its state transition matrix.

$$
y(t)=C(t) \Phi\left(t, t_{0}\right) x\left(t_{0}\right)+\int_{t_{0}}^{t} C(t) \Phi(t, \tau) B(\tau) u(\tau) d \tau+D(t) u(t)
$$

Since the state matrix $A$ is constant and considering the modal form of the equations of motion in Eq. (4), the state transition matrix is simply.

$$
\Phi\left(t, t_{0}\right)=e^{\Lambda\left(t-t_{0}\right)}
$$

This reveals that the eigenvalues of the structure measured using CSLDV are equal to the Floquet exponents of the LTP model. Hence, both the mode shapes and natural frequencies of the structure can be determined once the Floquet Exponents and time periodic mode shapes of the LTP system have been identified.

Werely [17] used Eq. (5) to extend the concept of a transfer function to linear time periodic systems. He derived the concept of a harmonic transfer function (HTF) that relates the input and output of a time-periodic system in a transfer function type manner. The primary difference is that, whereas a transfer function relates the output at a single frequency to the input at the same frequency, the HTF relates the input at a collection of frequencies to the output at that same comb of frequencies. Specifically, consider the Fourier transform of the response $y(t)$ shifted by $n \omega_{A}$,

$$
y_{n}(\omega)=\int_{-\infty}^{\infty} y(t) e^{\left(-j \omega-j n \omega_{A}\right) t} d t
$$

An EMP signal, denoted with bold uppercase in the following, is the collection of frequency shifted copies of the response. In the case of CSLDV measurements, there is only one measurement point so $y(t)$ is a scalar. Hence, the EMP representation is,

$$
\mathbf{Y}(\omega)=\left[\begin{array}{lllll}
\cdots & y_{-1} & y_{0} & y_{1} & \cdots
\end{array}\right]^{\mathrm{T}}
$$

The autospectrum of the EMP signal is found in the usual way,

$$
S_{y y}(\omega)=\mathrm{E}\left(\mathbf{Y}(\omega) \mathbf{Y}(\omega)^{H}\right)
$$

where $\mathrm{E}()$ denotes the expectation and ()$^{\mathrm{H}}$ denotes the Hermitian. Allen et al. showed in $[15,16]$ that the output autospectrum can be written as follows in terms of the modes of the state transition matrix,

$$
\left[S_{y y}(\omega)\right] \approx \sum_{r=1}^{N} \sum_{l=-\infty}^{\infty} \frac{\overline{\mathbf{C}}_{r, l} \mathbf{W}(\omega)_{r, l} \overline{\mathbf{C}}_{r, l}{ }^{H}}{\left[i \omega-\left(\lambda_{r}-i l \omega_{A}\right)\right]\left[i \omega-\left(\lambda_{r}-i l \omega_{A}\right)\right]^{H}}
$$

This has the same mathematical form as the output autospectrum of a multi-output linear time invariant system. Each denominator is multiplied by its complex conjugate, so the response is a sum of modal contributions squared. As with an LTI system, each numerator contains $\mathbf{W}(\omega)_{r, l}$, which can be shown to be the autospectrum of the net force exciting the $r$ th mode. 
There are also two notable differences between Eq. (10) and the equivalent expression for an LTI system. First, the expression contains a summation over both the modes, whose eigenvalues are $\lambda_{r}$, and also a summation over the harmonics of $\omega_{A}$ using the integer index $l$. Hence, the autospectrum of the LTP system will have peaks near each natural frequency, $\omega_{r}$, and also at the frequencies $\omega_{r} \pm l \omega_{A}$ for any integer $l$. Second, the mode vectors $\overline{\mathbf{C}}_{r, l}$ are not the usual collections of the mode shape at different points, but have a different definition. As discussed in [15, 16], the mode vectors $\overline{\mathbf{C}}_{r, l}$ are a collection of the Fourier coefficients of the $r$ th observed mode vector $C(t) \psi_{r}$ shifted by $l$. Specifically, $C(t) \psi_{r}$ is expanded in a Fourier series as follows

$$
C(t) \psi_{r}=\sum_{n=-\infty}^{\infty} C_{r, n} e^{j n \omega_{A} t}
$$

and then the Fourier coefficients are collected into $\overline{\mathbf{C}}_{r, l}$ after shifting by $l$.

$$
\overline{\mathbf{C}}_{r, l}=\left[\begin{array}{lllll}
\cdots & C_{r,-1-l} & C_{r,-l} & C_{r, 1-l} & \cdots
\end{array}\right]^{T}
$$

Here we shall refer to the autospectrum of the EMP signal as the harmonic power spectral density or HPSD. Since the HPSD has the same form as the PSD of a linear system, OMA techniques for LTI system scan be used to identify the modes of the system. However, one will obtain multiple estimates for each mode since each mode is present at a comb of frequencies $\omega_{r} \pm l \omega_{A}$. This can be addressed in a post processing step, but care must be taken since the mode vectors identified at each harmonic will not generally be of equal quality. Some of them will be strong and stand out above the noise while others might be weaker or contaminated by neighboring modes. A weighted average method can be employed to decrease the effect of the less reliable estimates. In addition, each of the estimates of $\overline{\mathbf{C}}_{r, l}$ can differ by a complex scale factor, so their phases should be aligned before averaging.

Theoretically, one must consider an infinite number of harmonics to characterize an LTP system, yet one would expect that most systems can be well approximated with a finite, perhaps even small number. When a range of harmonics, $n=-p \ldots p$, are used to describe the CSLDV output signal in eq. (7) and (8), the resulting auto spectrum matrix $S_{y y}(\omega)$ has the dimension of $\left[N_{p} \times N_{p} \times N_{f}\right]$, where $N_{p}=2 * p+1$ and $N_{f}$ is the number of frequency lines. One is free to use any of the rows (or columns) in the power spectrum matrix to identify the $\left[N_{p}\right.$ $\times 1$ vector $\overline{\mathbf{C}}_{r, l}$. In this work, only the power spectra along the primary column $S_{y y}(\omega)_{n, 0}=\mathrm{E}\left(\mathbf{Y}(\omega) y_{0}(\omega)^{H}\right)$, are used. Once a vector $\overline{\mathbf{C}}_{r, l}$ is found, one can reconstruct the time periodic mode shape of the system according to eq. (11) and then plot it against the laser path to generate the mode shape of the LTI system.

\section{Experimental Validation}

\subsection{Experimental Setup}

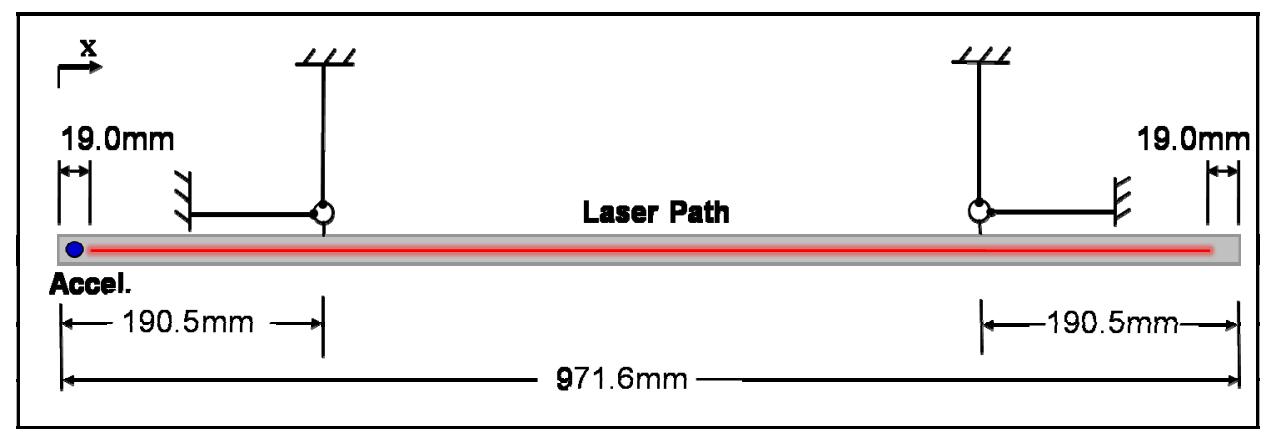

Figure 1: Schematic of test setup 
In order to validate the new OMA-CSLDV methodology, CSLDV was used to measure the modes of a free-free aluminum beam, as shown schematically in Figure 1 . The beam was suspended by soft bungee cables so the laser scan path would remain on the beam as it was excited. The supports were made as soft as possible and placed at the nodes of the first analytical bending mode to minimize the effect of the support stiffness on the beam's modes. A 5.2g accelerometer (PCB J351B11) was attached at the left end of the beam ( $x=6.3 \mathrm{~mm})$, the same configuration as in [13], so that the identified modes can be compared to the analytical model used in that previous work. The signal from the accelerometer was not used in any of the following.

Continuous-Scan Laser Doppler Vibrometry was employed to measure the beam's transient response using a Polytec $®$ PSV-400 scanning laser vibrometer with the customized mirror system described in [27]. To improve the laser signal strength and reduce noise, the front surface of the beam was covered with light weight retro-reflective tape (3M ScotchliteTM High Gain Reflective Sheeting 7610). The mirror system was used to scan the laser along the length of the beam sinusoidally at $1.5 \mathrm{~Hz}$ and $3 \mathrm{~Hz}$. The mirror driving voltage was manually adjusted until the laser traversed all but $19 \mathrm{~mm}$ from the ends of beam; this ensured that the laser remained on the beam throughout the tests. The beam was excited at random times and at random locations for 10 mins with an instrumented hammer (PCB 086C01), although the force signal from the hammer was not measured. A sampling frequency of $2560 \mathrm{~Hz}$ was selected to record the vibrometer signal, mirror driving signal and mirror output signal using a National Instruments PXI data acquisition system. To further reduce the noise, the vibrometer signal was filtered with a low-pass filter and down sampled to $1280 \mathrm{~Hz}$ before processing the measurements. This sampling frequency appeared to be sufficient to capture all of the frequency content that stood out above the noise floor.

\subsection{Validation}

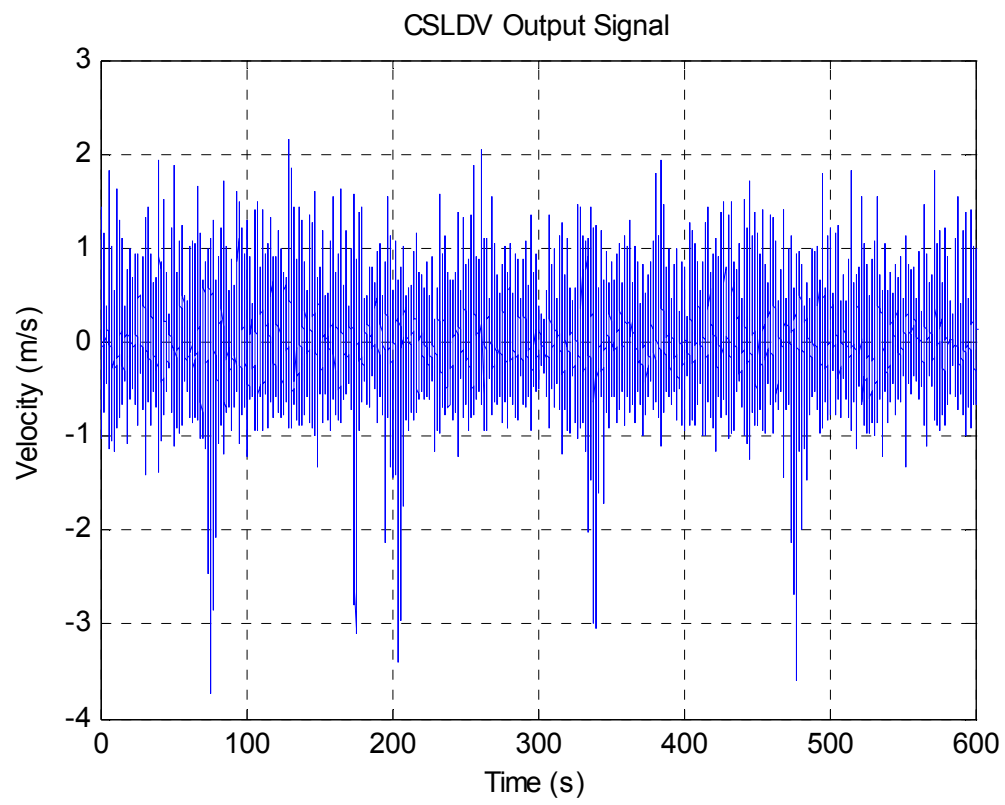

Figure 2: CSLDV output signal under random excitation $(1.5 \mathrm{~Hz}$ scan frequency)

Figure 2 shows the 10 minute long CSLDV output signal that was acquired at a scan frequency of $1.5 \mathrm{~Hz}$. The vibration signal appears to be random, although it drops to $-3 \mathrm{~m} / \mathrm{s}$ periodically during the measurement (the same phenomenon was noted when a $3 \mathrm{~Hz}$ scan frequency). This phenomenon seems to have been caused by the VD-03 velocity decoder used in the Polytec system, although its cause is not well understood. Measurements with the VD-08 decoder did not show this feature, but the largest range setting for that decoder was inadequate for this system. The output signal including these dropouts was used in the following analysis without any special treatment and fortunately, reasonable results were still obtained in spite of that contamination.

To form the EMP output signal $Y(\omega)$ in eq. (8), the CSLDV output signal was exponentially modulated with harmonics $n=-5 \ldots 5$ according to eq. (7). The number of harmonics required to accurately describe each mode shape was not known, but when low scan frequencies such as these are used, choosing a large number of harmonics may cause the harmonics from neighboring modes to overlap so that the mode shape cannot be accurately determined. Hence, one must choose the scan frequency carefully to avoid this difficulty. The authors 
have typically chosen the scan frequency to be large relative to the natural frequencies of interest in order to simplify matters $[12,28]$, although in this work smaller scan frequencies are explored since they make the spectra easier to interpret and reduce laser speckle noise [29, 30]. Other researchers, such as Stanbridge et al. typically use relatively small scan frequencies in their work, see [5-8] for examples.

The modulated CSLDV output signal was then decomposed into to 20 s long blocks to calculate $S_{y y}(\omega)$. A Hanning window was applied to each of the blocks with $75 \%$ overlap, resulting in 104 blocks for each modulated output signal. The auto and power spectra of the modulated output signal are then calculated on each block and averaged over all 104 blocks, resulting in a $[11 \times 11 \times 16385]$ harmonic power spectrum matrix. Recall that a single CSLDV signal was measured, but it was expanded into 11 signals so the Power Spectral Density (PSD) for this system is an 11 by 11 matrix at each frequency.

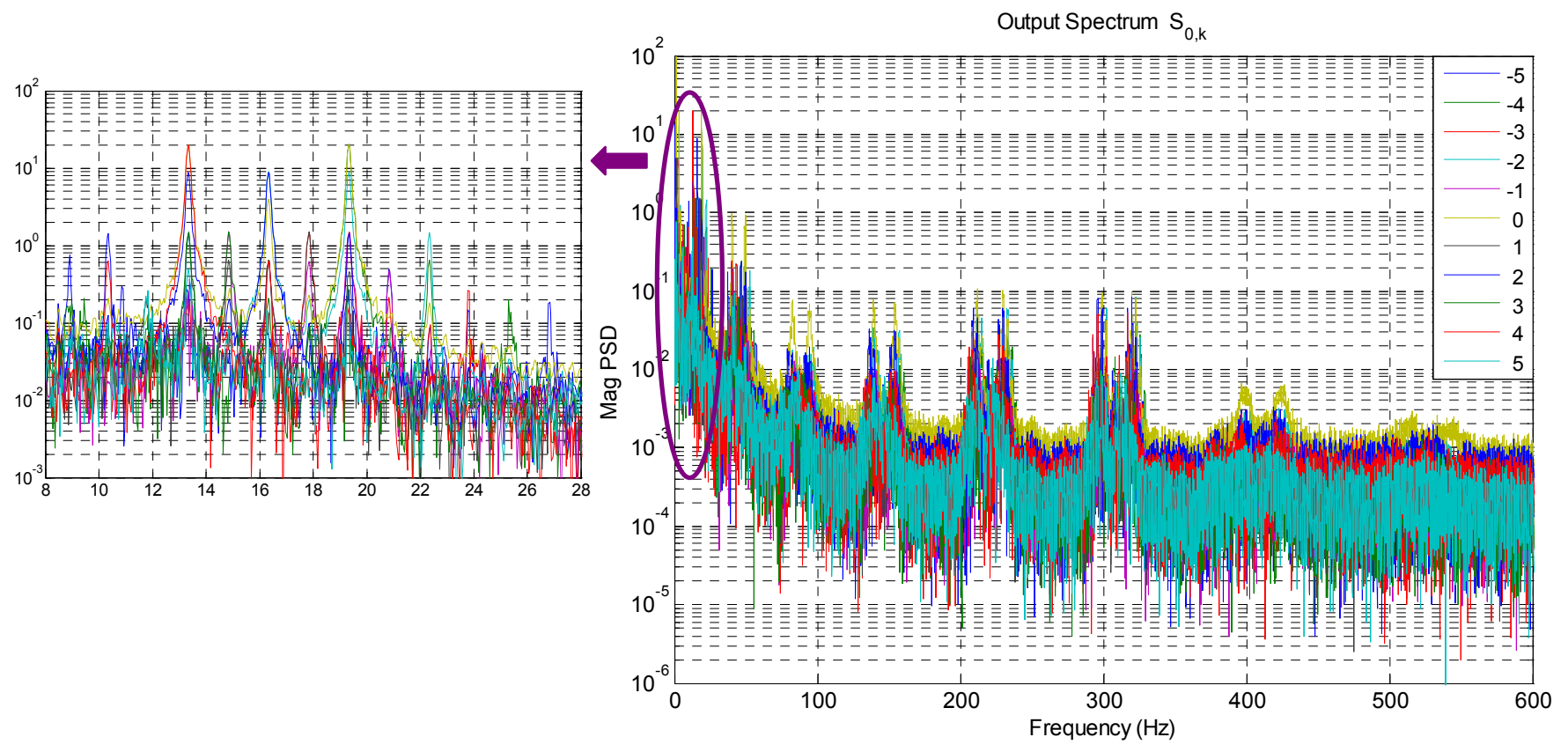

Figure 3: Harmonic PSD of CSLDV measurement at $1.5 \mathrm{~Hz}$ scan frequency. The pane on the left shows an expanded view of the measurement near $16 \mathrm{~Hz}$.

Figure 3 shows the power spectrum along the primary row of $S_{y y}(\omega)$. The eleven curves shown correspond to each of the $n$, $k$ th elements of $S_{y y}$ for $n=0$ and $k=-5 \ldots 5$. The spectra show clusters of strong harmonics around each of the natural frequencies. The view on the left focuses on the $1^{\text {st }}$ mode, whose natural frequency is $16.34 \mathrm{~Hz}$. Besides the peak at $16.34 \mathrm{~Hz}$, the $1^{\text {st }}$ mode is also responsible for the harmonics in the spectrum at $13.34 \mathrm{~Hz}, 14.85 \mathrm{~Hz}, 17.85 \mathrm{~Hz}, 19.34 \mathrm{~Hz}$, etc, all separated by multiples of $1.5 \mathrm{~Hz}$, which is the fundamental frequency $\omega_{A} /(2 \pi)$. The peak picking method can be used to identify the Fourier coefficients $\overline{\mathbf{C}}_{r, l}$ from the power spectra in Figure 3, and this approach was found to provide good accuracy in most cases. However, better results were obtained by fitting the measurements to single DOF modes using the Algorithm of Mode Isolation (AMI) [31]. This algorithm was modified from the version in [31] to fit squared modes to the power spectrum near each mode. The AMI algorithm identified the natural frequencies, damping ratios and mode shapes $\overline{\mathbf{C}}_{r, l}$ of the mode manifest at each of the peaks in the spectrum. 


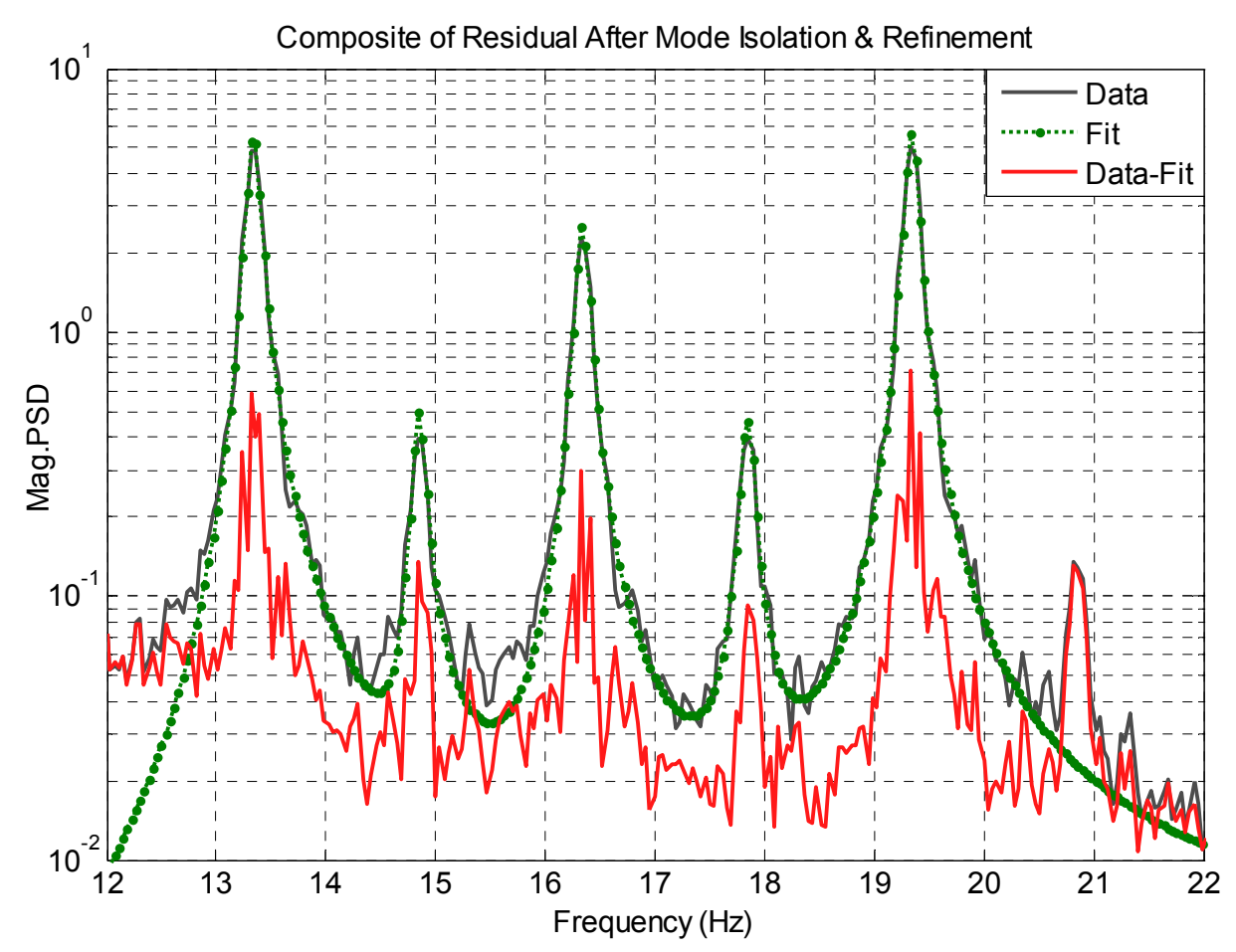

Figure 4: AMI fit of the 1 st mode using a $1.5 \mathrm{~Hz}$ scanning frequency

Figure 4 shows an AMI fit to several of the peaks arising from the $1^{\text {st }}$ mode, again for the $1.5 \mathrm{~Hz}$ scanning frequency. The gray solid line indicates the composite FRF, which is the average of the $11 \mathrm{FRFs}$ in 3 , and the green dash-dot line is the AMI fit curve. The 5 dominant harmonics of this mode are well approximated by the reconstructed spectrum. A third curve shows the composite of the difference between the measurement and the reconstruction, revealing that the fit is superior at the three dominant peaks. The response stands out above the noise (due to imperfection in the LDV and to the non-flatness of the input spectrum) by about one order of magnitude. Table 1 shows the normalized Fourier coefficient vectors identified from the 5 harmonics shown in Figure 4. Each of the identified coefficient vectors should contain the same Fourier coefficients $C_{r, n}$, but those coefficients are shifted by $l$ in each of the identified vectors $\overline{\mathbf{C}}_{r, l}$ and each of those vectors may have a different complex scale factor. The scale differences are addressed by normalizing the vector by dividing by the largest value and then rotating the vector to the same phase so that they can be averaged. To minimize the noise, the largest value in each identified Fourier coefficient vector $\overline{\mathbf{C}}_{r, l}$ is used as the weight during the averaging. Some higher order Fourier coefficients have large discrepancy because of the noise (see $C_{1,5} C_{1,6}$ and $C_{1,7}$ in Table 1), but those terms are from weakly represented peaks in the spectrum and so they become much smaller applying the weighted average.

Table 1 Shifted Fourier coefficient vectors of the 1 st mode under $1.5 \mathrm{~Hz}$ scanning frequency

\begin{tabular}{c|c|ccccc}
\hline & Averaged & \multicolumn{5}{|c}{ Harmonics of ${ }^{\text {st }}$ mode } \\
\hline Frequency & 16.3391 & 13.3372 & 14.8429 & 16.3349 & 17.8412 & 19.3392 \\
\hline Weight & 1 & 0.3629 & 0.0275 & 0.159 & 0.0296 & 0.3536 \\
\hline$r / l$ & $\overline{\mathbf{C}}_{1,0}$ & $\overline{\mathbf{C}}_{1,2}$ & $\overline{\mathbf{C}}_{1,1}$ & $\overline{\mathbf{C}}_{1,0}$ & $\overline{\mathbf{C}}_{1,-1}$ & $\overline{\mathbf{C}}_{1,-2}$ \\
\hline$C_{1,-7}$ & $-0.0058-0.0013 \mathrm{i}$ & $0.0080-0.0004 \mathrm{i}$ & 0 & 0 & 0 & 0 \\
$C_{1,-6}$ & $-0.0259+0.0087 \mathrm{i}$ & $-0.0083+0.0055 \mathrm{i}$ & $-0.0490-0.0095 \mathrm{i}$ & 0 & 0 & 0 \\
$C_{1,-5}$ & $-0.0030+0.0121 \mathrm{i}$ & $0.0104-0.0119 \mathrm{i}$ & $-0.0000-0.0027 \mathrm{i}$ & $0.0104-0.0061 \mathrm{i}$ & 0 & 0 \\
\hline
\end{tabular}




\begin{tabular}{l|c|ccccc}
\hline$C_{1,-4}$ & $-0.0779+0.0123 \mathrm{i}$ & $0.0536-0.0032 \mathrm{i}$ & $0.0301+0.0034 \mathrm{i}$ & $0.0664-0.0029 \mathrm{i}$ & $0.0163-0.0639 \mathrm{i}$ & 0 \\
$C_{1,-3}$ & $0.0049-0.0050 \mathrm{i}$ & $-0.0055+0.0087 \mathrm{i}$ & $0.1186+0.0559 \mathrm{i}$ & $-0.0011+0.0067 \mathrm{i}$ & $-0.0043+0.0200 \mathrm{i}$ & $-0.0065+0.0134 \mathrm{i}$ \\
$C_{1,-2}$ & 1 & $-0.9946-0.1034 \mathrm{i}$ & $-0.9358-0.0859 \mathrm{i}$ & $-0.9943-0.1063 \mathrm{i}$ & $-0.9944-0.1059 \mathrm{i}$ & $-0.9945-0.1051 \mathrm{i}$ \\
$C_{1,-1}$ & $-0.0729-0.0034 \mathrm{i}$ & $0.0689+0.0082 \mathrm{i}$ & $0.1461+0.0457 \mathrm{i}$ & $0.0709+0.0069 \mathrm{i}$ & $0.0421-0.0036 \mathrm{i}$ & $0.0759+0.0083 \mathrm{i}$ \\
$C_{1,0}$ & $0.4464+0.0771 \mathrm{i}$ & $-0.4211-0.1139 \mathrm{i}$ & $-0.4045-0.1223 \mathrm{i}$ & $-0.4267-0.1196 \mathrm{i}$ & $-0.3882-0.1069 \mathrm{i}$ & $-0.4232-0.1175 \mathrm{i}$ \\
$C_{1,1}$ & $-0.0769-0.0185 \mathrm{i}$ & $0.0735+0.0217 \mathrm{i}$ & $0.1239+0.0214 \mathrm{i}$ & $0.0723+0.0228 \mathrm{i}$ & $0.1089+0.0242 \mathrm{i}$ & $0.0727+0.0196 \mathrm{i}$ \\
$C_{1,2}$ & $0.9354+0.3048 \mathrm{i}$ & $-0.8994-0.3967 \mathrm{i}$ & $-0.9121-0.4099 \mathrm{i}$ & $-0.9077-0.3987 \mathrm{i}$ & $-0.8801-0.3941 \mathrm{i}$ & $-0.9120-0.4074 \mathrm{i}$ \\
$C_{1,3}$ & $0.0114+0.0180 \mathrm{i}$ & $-0.0058-0.0178 \mathrm{i}$ & $-0.0707-0.0498 \mathrm{i}$ & $-0.0107-0.0149 \mathrm{i}$ & $-0.0337-0.0465 \mathrm{i}$ & $-0.0082-0.0174 \mathrm{i}$ \\
$C_{1,4}$ & $-0.0346-0.0188 \mathrm{i}$ & 0 & $-0.0186+0.0253 \mathrm{i}$ & $0.0533+0.0378 \mathrm{i}$ & $0.0645+0.0436 \mathrm{i}$ & $0.0519+0.0333 \mathrm{i}$ \\
$C_{1,5}$ & $-0.0038-0.0009 \mathrm{i}$ & 0 & 0 & $0.0074+0.0037 \mathrm{i}$ & $0.0054-0.0027 \mathrm{i}$ & $0.0040+0.0023 \mathrm{i}$ \\
$C_{1,6}$ & $0.0006+0.0016 \mathrm{i}$ & 0 & 0 & 0 & $-0.0248+0.0059 \mathrm{i}$ & $0.0000-0.0073 \mathrm{i}$ \\
$C_{1,7}$ & $-0.0009-0.0001 \mathrm{i}$ & 0 & 0 & 0 & 0 & $0.0023-0.0019 \mathrm{i}$ \\
\hline
\end{tabular}

The mode shapes reconstructed from these Fourier coefficient vectors are plotted in Figure 5, as well as the averaged mode shape from the weighted average of all of the Fourier coefficients. It can be seen that the mode shapes from the -1 and 1 harmonics have some discrepancy from the ones from the $-2,0$ and 2 harmonics. This is to be expected since the -1 and 1 harmonics have about 10 times smaller amplitude in Figure 4 , so they are much more strongly influenced by noise. Any of the other harmonics gives a reasonable estimate of this mode's shape.

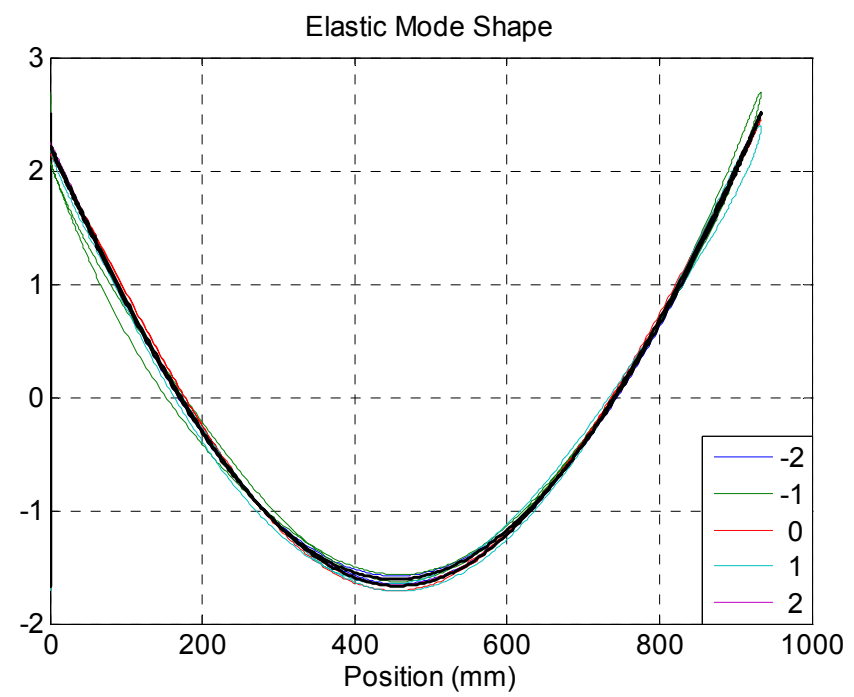

Figure 5: Mode shapes reconstructed from each of the five groups of harmonics in Table 1. The solid black line shows the mode shape computed from the weighted average of the harmonics.

All of the other modes were identified in a similar manner. Table 2 shows the natural frequencies of each of the modes that were identified from the harmonic spectra in Figure 6 . The $1.5 \mathrm{~Hz}$ scan frequency was used for the first two modes, while the rest were extracted from the measurements with the $3 \mathrm{~Hz}$ scan frequency. With the higher modes it was possible to compute the harmonic autospectrum for $n=-8: 8$, so that was used for modes 3 through 7. The value in the column labeled "OMA" is the average of the natural frequency estimated at each of the harmonics, after shifting each by an integer multiple of $\omega_{A}$. The standard deviation of the natural frequency estimates is also shown, which is very small in all cases, revealing that each of the harmonics gives a very similar estimate of the natural frequency. The values in the column "Analytical" are the natural frequencies of a tuned analytical model, which is an Euler-Bernoulli beam model that was tuned to account for the mass of the accelerometer as described in [13]. All of the OMA identified natural frequencies agree well with the analytical values, the largest difference is a few $\mathrm{Hz}$ for the higher modes, but the analytical model was found to be somewhat inaccurate for these modes so the OMA result is still within the margin of error for the analytical model. 
Table 2 Comparison of analytical and experimental modes

\begin{tabular}{ccccccc}
\hline No. & $\begin{array}{c}\text { Analytical } \\
\text { Freq }(\mathrm{Hz})\end{array}$ & $\begin{array}{c}\text { OMA } \\
\text { Freq. }(\mathrm{Hz})\end{array}$ & Std & $n$ & $\begin{array}{c}\text { Scan } \\
\text { Frequency }\end{array}$ & MAC \\
\hline 1 & 16.38 & 16.34 & 0.01 & $-5: 5$ & 1.5 & 1.00 \\
2 & 45.35 & 45.15 & 0.03 & $-5: 5$ & 1.5 & 0.98 \\
3 & 89.23 & 88.73 & 0.10 & $-8: 8$ & 3 & 0.95 \\
4 & 147.99 & 146.99 & 0.02 & $-8: 8$ & 3 & 0.98 \\
5 & 221.71 & 221.07 & 0.01 & $-8: 8$ & 3 & 0.95 \\
6 & 310.49 & 309.53 & 0.01 & $-8: 8$ & 3 & 0.92 \\
7 & 414.37 & 411.57 & 0.10 & $-8: 8$ & 3 & 0.92 \\
\hline
\end{tabular}

Figure 6 shows the mode shapes of each of the identified modes, as well as the shapes from the tuned analytical model from [13]. The mode shapes agree very well with the analytical model, except for the $3^{\text {rd }}$ and $7^{\text {th }}$ modes which show significant discrepancies. The $3^{\text {rd }}$ mode was heavily damped and the harmonics overlapped with each other even when using a $3 \mathrm{~Hz}$ scanning frequency, so it was difficult to identify. The $7^{\text {th }}$ mode was weakly excited since the hammer force had rolled off significantly near its natural frequency, so it did not stand out sufficiently above the noise. This mode was also difficult to identify using the authors original CSLDV approach, as described in [12]. The MAC values between the analytical mode shapes and the CSLDV mode shapes are listed in Table 2; the worst MAC values occur for the $6^{\text {th }}$ and $7^{\text {th }}$ modes, but those values are still above 0.92 .
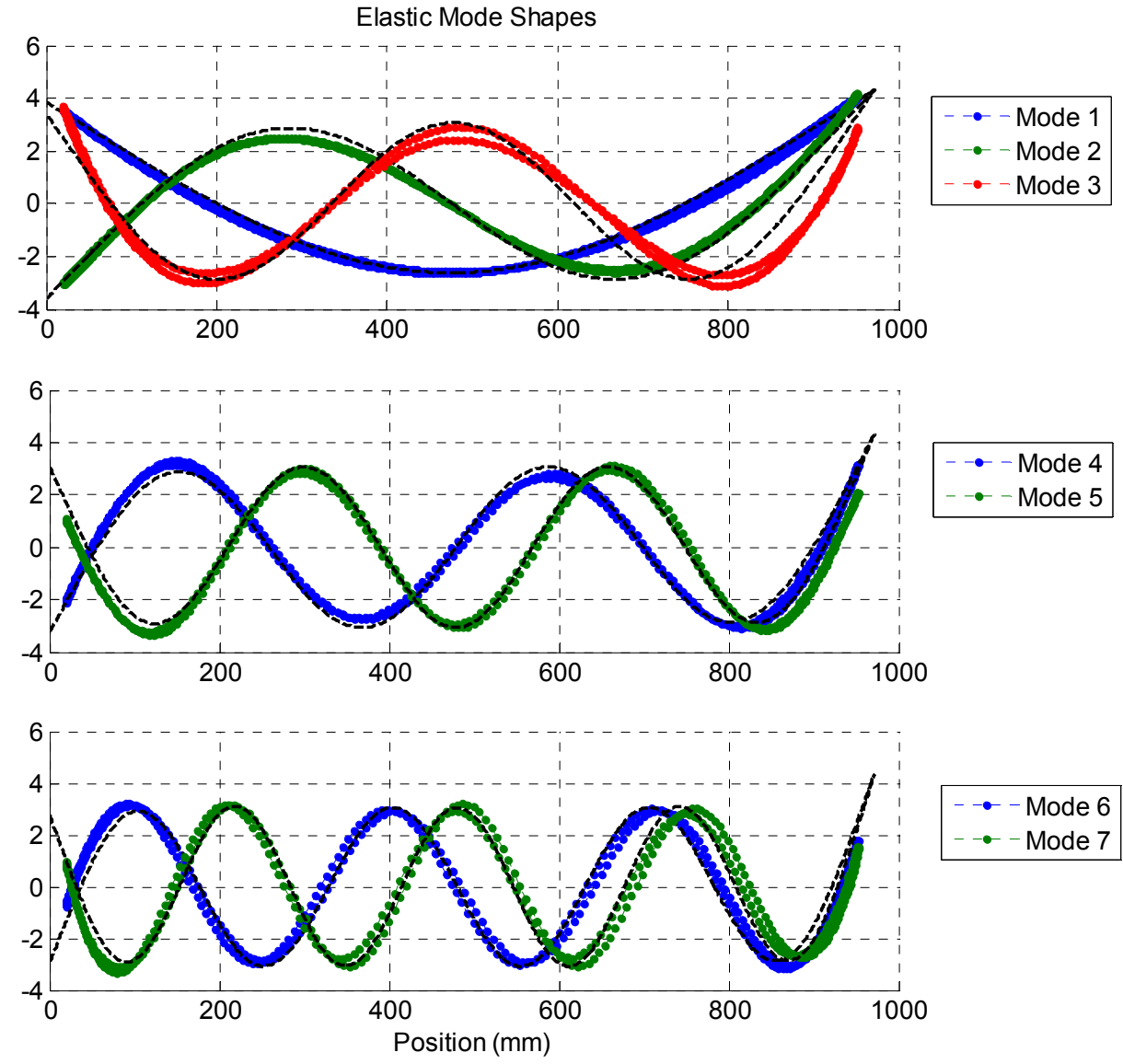

Figure 6 Mode shapes identified by CSLDV at 1.5Hz and $3 \mathrm{~Hz}$ scanning frequencies. Solid black lines denote the analytical shapes; dots show the shapes from OMA 


\subsection{Effect of Scan Frequency}

As these results illustrate, one must take some care to choose an acceptable scan frequency. As mentioned previously, low scan frequencies were chosen in this work so that the harmonics of each mode would occur in a cluster, as illustrated in Figure 3. However, this may lead to difficulty if many harmonics are needed to describe a mode, since the harmonics from one mode may overlap with those from the neighboring modes, especially when the modes are close to each other. In addition, if one mode is heavily damped, the scanning frequency must be large to distinguish the harmonics belonging to this mode. Figure 7 demonstrates this issue by comparing the spectrum near the heavily damped $3^{\text {rd }}$ mode for $1.5 \mathrm{~Hz}$ and $3 \mathrm{~Hz}$ scanning frequencies. The harmonics of this mode overlap significantly when a $1.5 \mathrm{~Hz}$ scanning frequency was used. Though one can still identify the natural frequency and mode shape from the strongest harmonics, the mode shape is somewhat contaminated by the other nearby harmonics. A better estimate of the mode shape is obtained with a $3 \mathrm{~Hz}$ scanning frequency. Its harmonics are better separated so the Fourier coefficients estimated from each peak are not as strongly affected by the neighboring peaks.
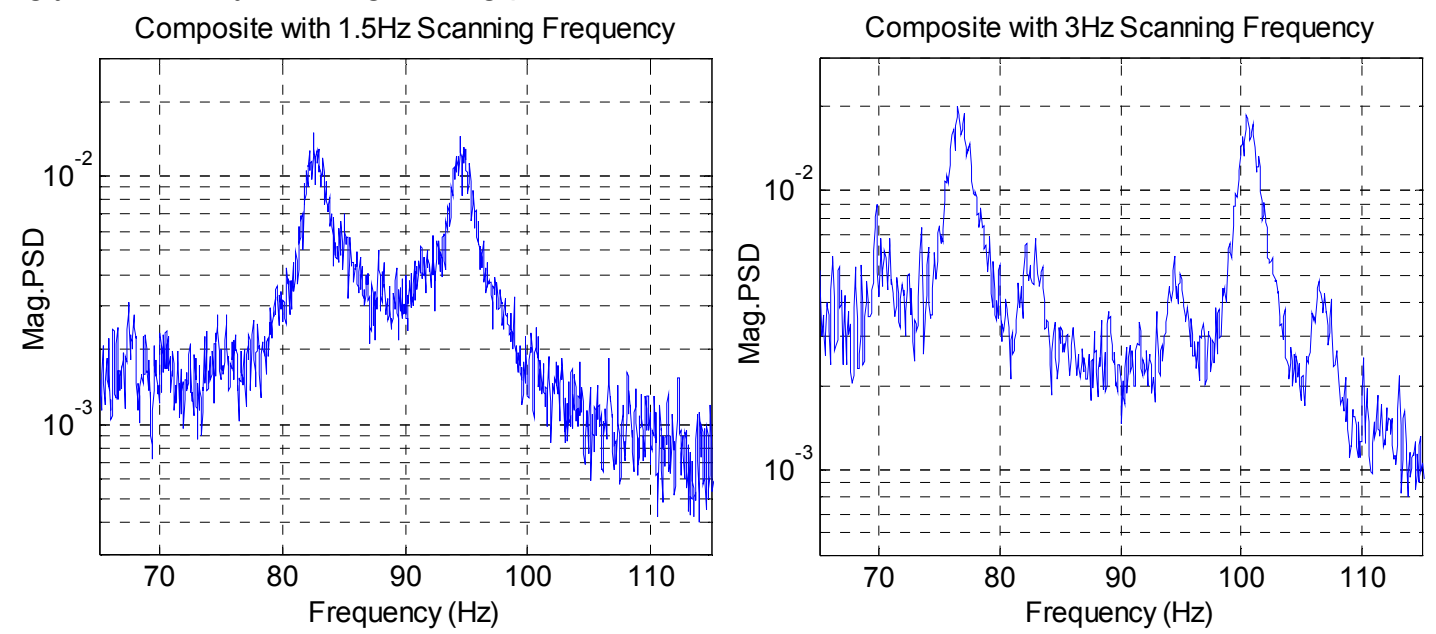

Figure 7: Composites of the harmonic PSD near the $3^{\text {rd }}$ mode with $1.5 \mathrm{~Hz}$ (left) and $3 \mathrm{~Hz}$ (right) scanning frequencies.

The most straight forward approach for selecting the scan frequency is to choose one that is higher than the highest mode that is excited. This assures that all of the harmonics in eq. (10) occur at distinct frequencies. Unfortunately, this may result in excessive noise with current lasers and for some systems the scan frequency needed may even exceed the capabilities of the mirror system. On the other hand, CSLDV is most advantageous for systems that have low natural frequencies and hence require long time records, so limitations of the laser and mirror system may not be a significant concern. A high scan frequency produces a spectrum that is a little more complicated than those shown above, since each of the mode peaks may be present in each frequency band for which $n \omega_{\mathrm{A}}<\omega<(n+1) \omega_{\mathrm{A}}$. This makes the spectra more difficult to interpret visually. In previous works the authors circumvented this difficulty by lifting the measurements, which effectively aliases the CSLDV signal producing a collection of signals that are limited to $0<\omega<\omega_{\mathrm{A}} / 2$ [12]. An analog of this method has not yet been developed for autospectra, so in this work we have worked with the spectra directly. 


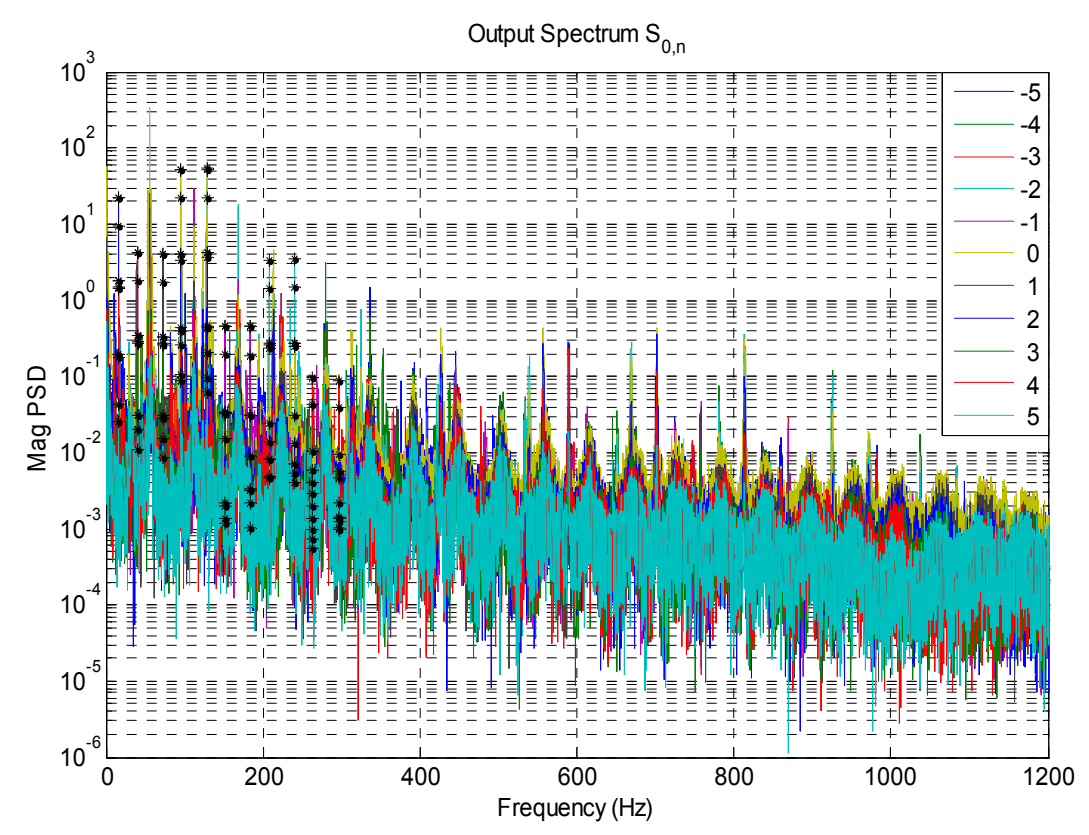

Figure 8: Harmonic PSD of CSLDV measurement at $56 \mathrm{~Hz}$ scan frequency

Figure 8 gives an example of the measurement under $56 \mathrm{~Hz}$ scanning frequency, where the black stars indicate the harmonics of the first mode $\left(f_{n}=16.34 \mathrm{~Hz}\right)$. The harmonics of this mode no longer appear in a cluster as in Figure 3 but throughout the band for $16.34 \mathrm{~Hz} \pm n 56 \mathrm{~Hz}$. Some of the harmonics for negative $n$ fold about zero frequency occurring at positive frequencies $|16.34 \mathrm{~Hz}-n 56 \mathrm{~Hz}|$, complicating matters further. Nevertheless, the same identification procedure can still be applied as long as the harmonics of each mode can be located. When reconstructing the mode shapes, one should note that the folded harmonics actually correspond to positive harmonics of the complex conjugate eigenvalue, so the identified vectors $\overline{\mathbf{C}}_{r, l}$ will be complex conjugates and have the Fourier coefficients in opposite order of those identified from the positive harmonics.

Figure 9 shows the mode shapes that were identified from the power spectrum in Figure 8 . The peak picking method was used to obtain the Fourier coefficient vector at each harmonic instead of AMI in this case. The reconstructed mode shapes still show great agreement with the analytical ones. However, the grid on which the mode shapes is plotted is coarser to reflect the fact that the $2560 \mathrm{~Hz}$ sampling rate only produces 46 samples per period at a $56 \mathrm{~Hz}$ scan frequency measurement. A higher scan frequency could have been used to obtain a finer measurement grid, or if one is certain that all of the important Fourier Coefficients have been obtained then one could reconstruct the mode shapes with any desired spatial resolution [6]. 

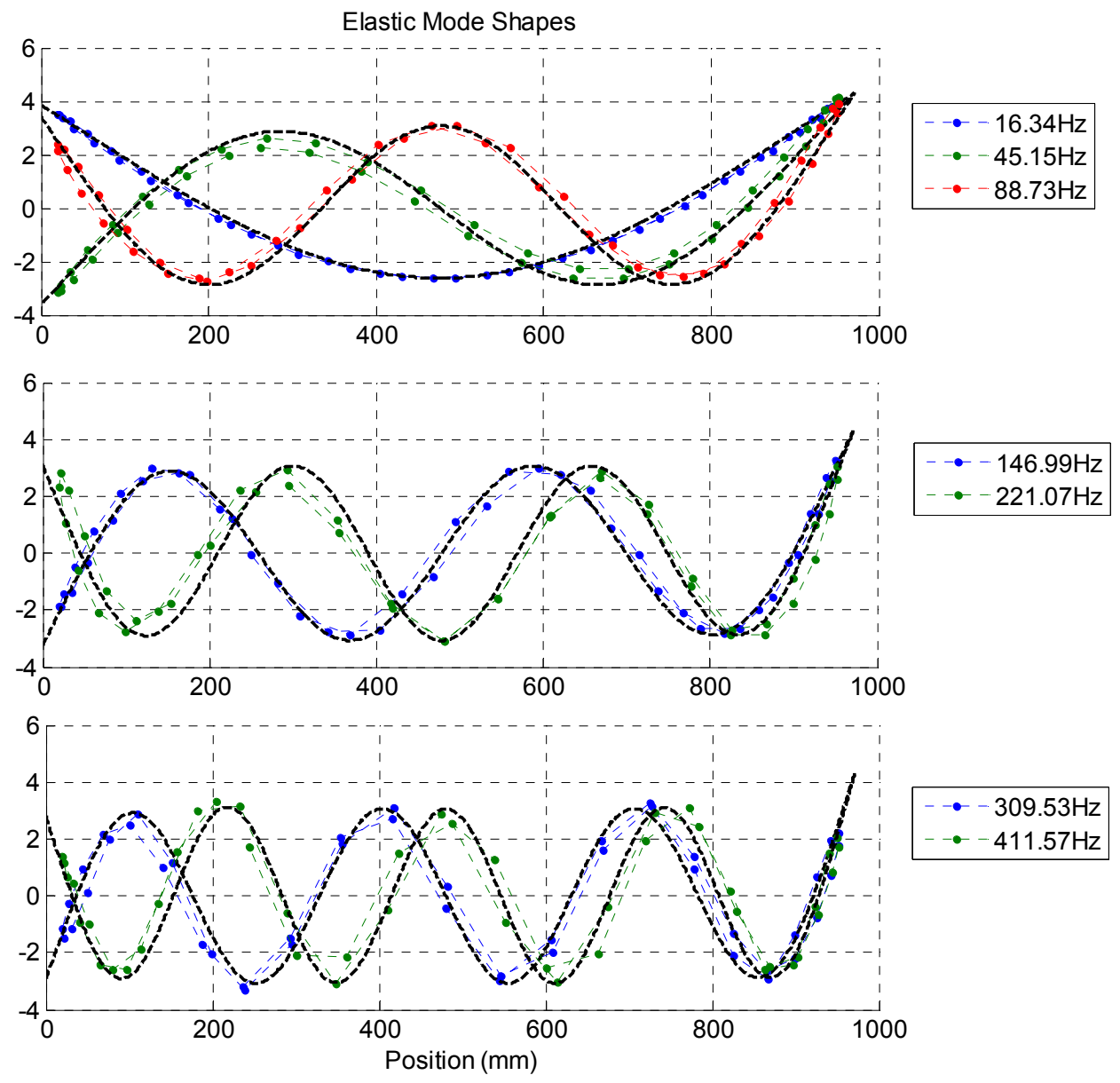

Figure 9: Mode shapes identified by CSLDV at 56Hz scanning frequency. Solid black lines denote the analytical shapes; dots show the shapes identified by OMA-CSLDV. 


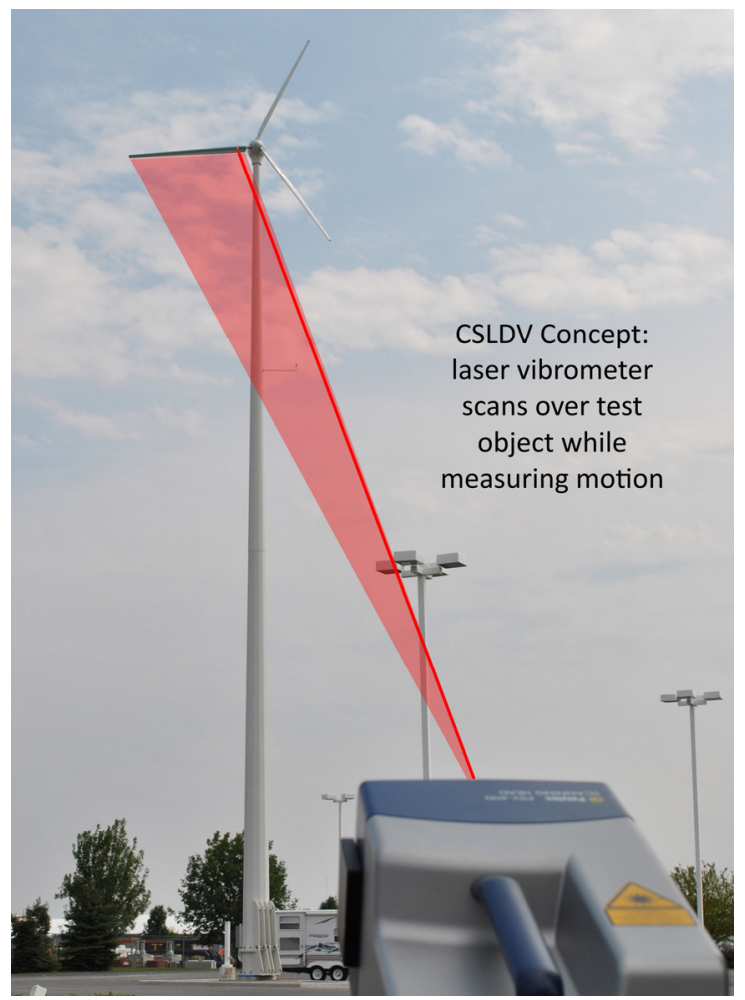

Figure 10: Photograph from CSLDV test of a wind turbine blade with a graphic illustrating the scan pattern.

The proposed method was applied to identify the natural frequencies and mode shapes of a wind turbine blade as depicted in Figure 10. The blade is one of three that comprise the turbine rotor. For all of the tests described here, the turbine rotor was locked by applying the brake and the blade of interest was pitched so that the laser was nominally perpendicular to the chord of the blade (i.e. measuring in the flapwise direction). The laser scanned over as much as possible of the $4.3 \mathrm{~m}$ long blade. For the results shown here the laser was $66 \mathrm{~m}$ from the base of the tower and the tower heights is $30 \mathrm{~m}$, so the total distance from the laser head to the blade is approximately $72.5 \mathrm{~m}$. The same retro-reflective tape was applied on the blade to increase the signal strength. This tape was necessary to obtain any signal at all from the vibrometer, and the increased reflectivity made the laser spot easy to locate when it was on the tape. The blade was excided purely by the wind, whose maximum speed was measured to be $3.5-3.75 \mathrm{~m} / \mathrm{s}$ at the conclusion of testing, by a sensor mounted on the tower and which can be seen in the figure about two-blade lengths down from the top of the tower.

Before acquiring CSLDV measurements, the laser spot was fixed near the tip of the blade and the power spectrum was estimated using the LDV software to compute the PSD over about a 10 minute period. The power spectrum is shown in Figure 11. At least seven peaks are seen in the spectrum, each of which is presumed to correspond to a natural frequency of the system. The frequencies of each of these peaks are listed in Table 3. Beyond about $25 \mathrm{~Hz}$ the wind apparently does not excite the system significantly as the measurement seems to reduce to noise above that frequency. One should note that the seven modes seen in Figure 11 are not expected to be seven bending modes of a simple beam. The turbine is comprised of three nominally identical blades so each blade bending mode occurs three times, at two or three distinct frequencies depending on the stiffness properties of the tower and nacelle. 


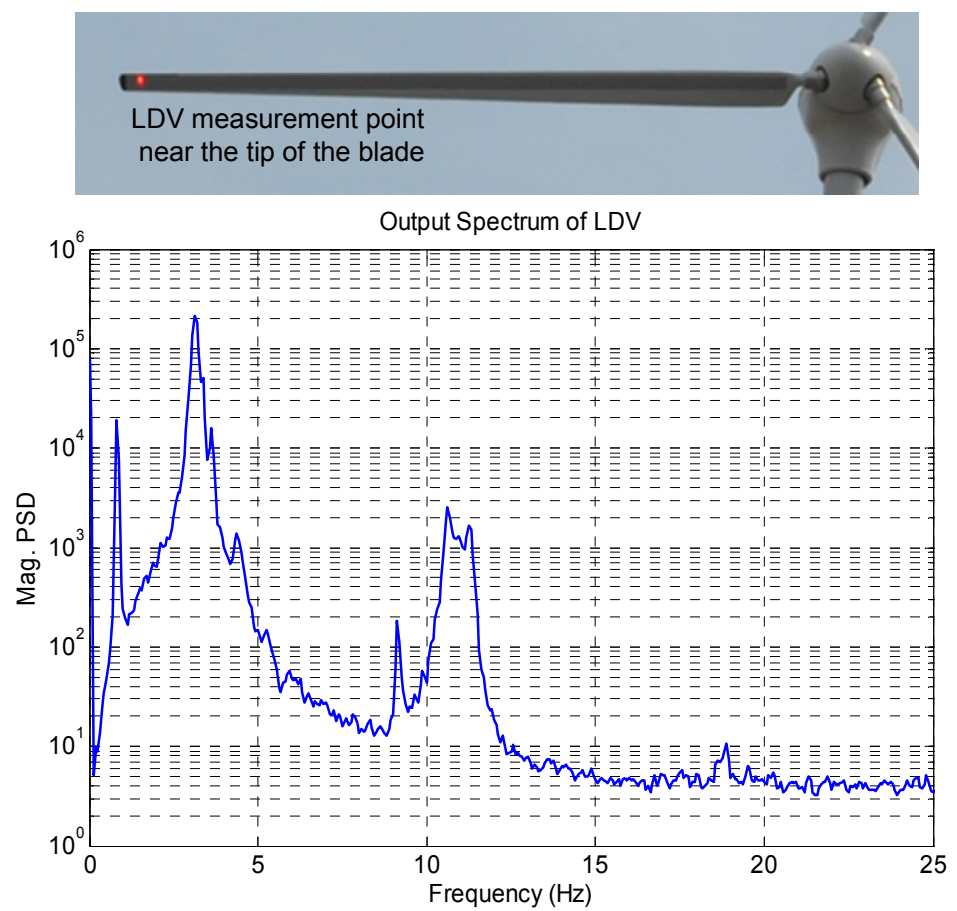

Figure 11: PSD of LDV output signal with the laser measuring at a fixed point near the tip of the blade

The LDV was then set to continuously scan the blade for $10 \mathrm{~min}$ at a time at several different scan frequencies ranging from $0.8 \mathrm{~Hz}$ to $9.3 \mathrm{~Hz}$. Both low and relatively high frequencies were investigated. The sampling frequency for these tests was $2560 \mathrm{~Hz}$, which was sufficient to capture the highest mode excited by the wind even at the $9.3 \mathrm{~Hz}$ scanning frequency.

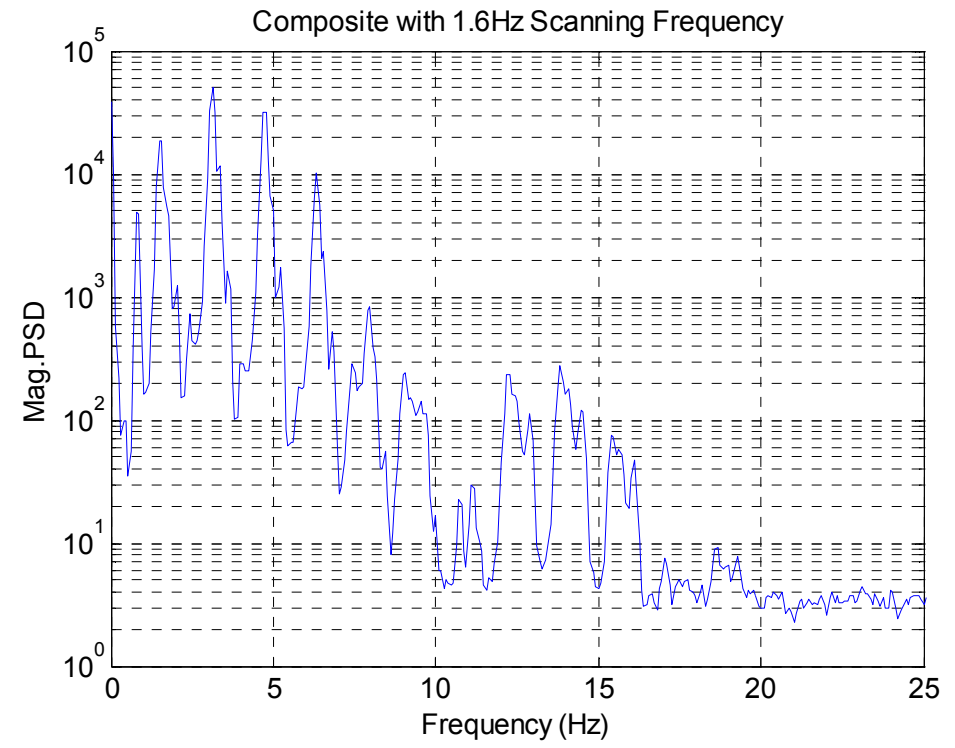

Figure 12: Composite of harmonic PSD of the CSLDV measurement with $1.6 \mathrm{~Hz}$ scanning frequency

Figure 12 shows the power spectra of the modulated CSDLV output signal using a $1.6 \mathrm{~Hz}$ scanning frequency. The harmonics $n=-3 \ldots 3$ were used to create the harmonic PSD. As was also the case in the signal from the fixed test, the spectra seems to reduce to noise above about $25 \mathrm{~Hz}$. The harmonics of several modes are visible in the spectrum, but it is difficult to determine which harmonics go with each natural frequency since they are all so closely spaced. Therefore, the point measurement in Figure 11 was used to determine which frequencies to search for each of the system's modes. The natural frequencies thus identified in the harmonic spectra are listed in Table 3, along with the seven frequencies identified from the tip spectrum in Figure 11. Also 
listed are the first 2 bending modes obtained by a conventional hammer-accelerometer test on a similar blade when it was mounted in a stiff fixture on the ground.

The mode shapes constructed from the harmonic power spectra are shown in Figure 13, found by computing the weighted average of the Fourier coefficients identified by the peak picking method at each of the significant harmonics. The mode shapes reveal that the mode at $0.81 \mathrm{~Hz}$ is predominantly a tower bending mode, where the tower bends and the blade moves as a rigid body. As reported in the table, the first bending mode of a single blade in a stiff fixture was $3.36 \mathrm{~Hz}$, which is close to the frequency of the next three modes identified by OMA at $3.13,3.36$ and $3.62 \mathrm{~Hz}$. Each of these modes shows the blade bending with the familiar shape reminiscent of the first mode of a cantilever beam. However, the mode shape of the $3.62 \mathrm{~Hz}$ mode seems to show some displacement at the root of the blade. This would seem reasonable since the symmetric flapwise mode of a horizontal axis wind turbine such as this tends to occur at higher frequency than the yaw and tilt flapwise modes (see, e.g. [32, 33]). The mode seen at $4.38 \mathrm{~Hz}$ in the tip measurement was not identifiable in the harmonic autospectrum, so its mode shape could not be determined. The first edgewise mode of this blade occurred at $5.24 \mathrm{~Hz}$ when the blade was mounted in the stiff fixture, so this peak in the tip spectrum may come about due to edgewise motion of the blade and the fact that the blade twists from root to tip. A $9.13 \mathrm{~Hz}$ mode is also seen in the fixed point LDV test and not in the CSLDV test. It may have been buried in the harmonics of the other modes or may simply be poorly excited in the CSLDV test. The last three modes that are identified have shapes that agree very well with the second bending mode of a cantilevered beam, and their frequencies at $10.63 \mathrm{~Hz}, 10.86 \mathrm{~Hz}$ and $11.29 \mathrm{~Hz}$ are similar to that of the blade in the fixture. All of these results were obtained from the CSLDV measurements at the $1.6 \mathrm{~Hz}$ scan frequency. Similar results were obtained at some of the other scan frequencies.

Table 3: Natural frequencies of wind turbine blade

\begin{tabular}{cccc}
\hline Mode & $\begin{array}{c}\text { Conventional test } \\
\text { in stiff fixture }\end{array}$ & $\begin{array}{c}\text { Fixed point OMA } \\
\text { on tower }\end{array}$ & $\begin{array}{c}\text { CSLDV OMA } \\
\text { on tower }\end{array}$ \\
\hline- & - & $0.81 \mathrm{~Hz}$ & $0.78 \mathrm{~Hz}$ \\
Flap Wise & & $3.13 \mathrm{~Hz}$ & $3.13 \mathrm{~Hz}$ \\
Bending 1 & $3.36 \mathrm{~Hz}$ & $3.37 \mathrm{~Hz}$ & $3.36 \mathrm{~Hz}$ \\
Edge Wise & & $3.63 \mathrm{~Hz}$ & $3.62 \mathrm{~Hz}$ \\
Bending 1 & $5.24 \mathrm{~Hz}$ & $4.38 \mathrm{~Hz}$ & - \\
- & - & $9.13 \mathrm{~Hz}$ & - \\
Flap Wise & & $10.63 \mathrm{~Hz}$ & $10.62 \mathrm{~Hz}$ \\
Bending 2 & $11.40 \mathrm{~Hz}$ & $10.94 \mathrm{~Hz}$ & $10.86 \mathrm{~Hz}$ \\
\hline
\end{tabular}



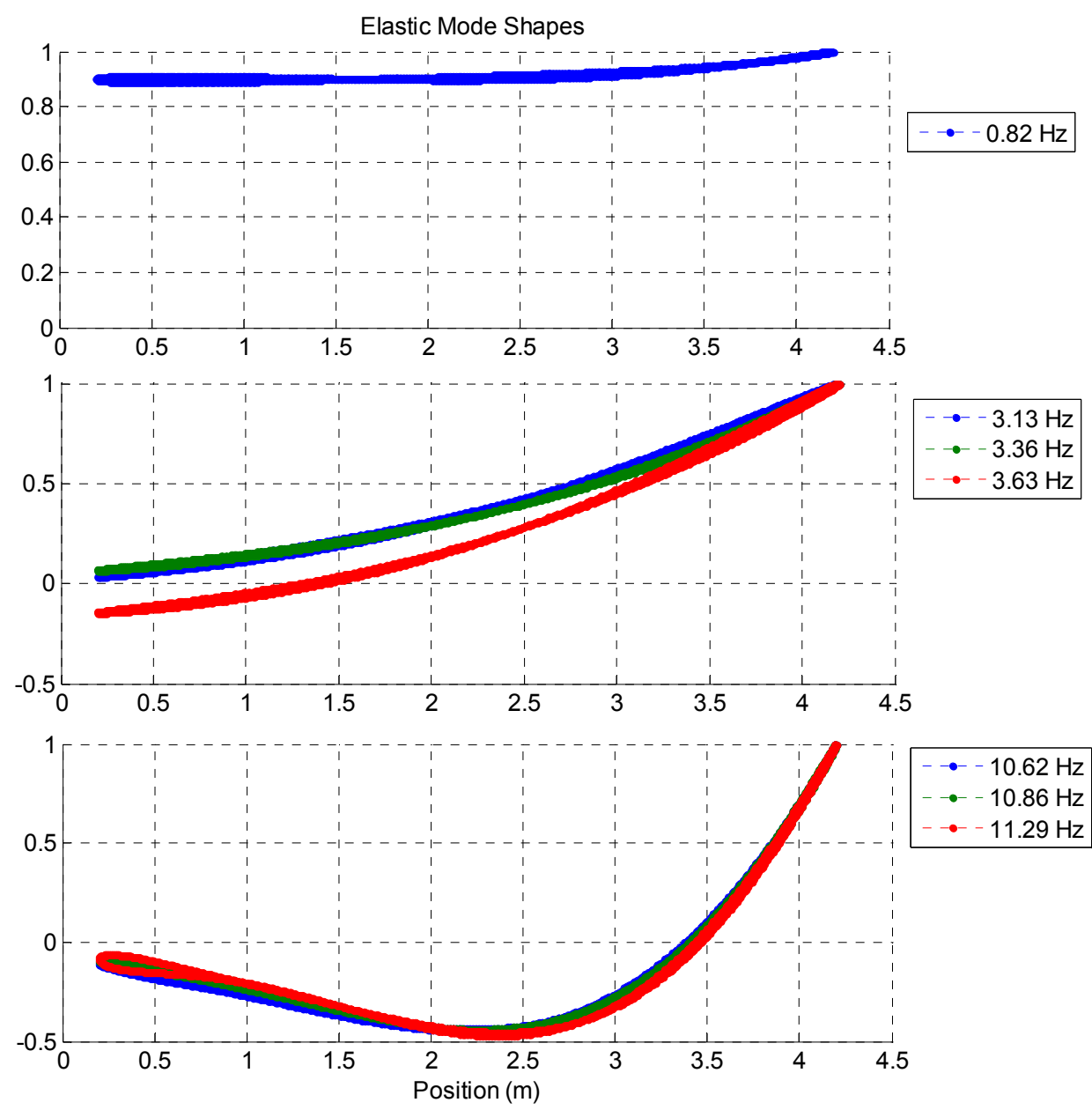

Figure 13: Mode shapes of wind turbine blade identified by CSLDV at 1.6Hz scanning frequency

\section{Conclusions}

This paper has extended Continuous Scanning Laser Doppler Vibrometry to output only measurements (OMA-CSLDV) by capitalizing on a recently developed system identification methodology for linear time-periodic systems. As with conventional OMA, the method assumes that the forces exciting the system are random, white and that they sufficiently excite all of the modes of interest. The theoretical development reveals that when CSLDV is used, each mode appears at several peaks in the power spectrum. The measured CSLDV signal is exponentially modulated to create what is called a harmonic power spectrum, and then a standard OMA algorithm such as peak-picking can be used to identify the modes of the system. Each identified mode is comprised of a set of Fourier coefficients that describe the variation of the mode shape as a function of time as the laser scans periodically. In this work, the harmonic power spectra were processed both by peak-picking and by curve fitting using a least squares algorithm (the Algorithm of Mode Isolation). Both methods gave good results for the systems studied here.

The new OMA-CSLDV methodology was first validated on a free-free beam in a laboratory setting. OMACSLDV was found to give results that were very similar to those obtained by the authors' hammer excited CSLDV method, identifying spatially detailed mode shapes of the first seven modes of the beam from one time record. Various scan frequencies were investigated and the associated issues were discussed. The output spectra shown here seemed to be considerably noisier than the spectra of the free-response that was processed in the previous work [13], but the same has been observed when comparing conventional OMA with EMA methods where the input forces are measured and controlled.

The methodology presented here was further studied by using it to measure the modes of a parked wind turbine. The vibrometer was used to measure the spectrum of the response at the tip of the blade (conventional 
fixed-point OMA) and CSLDV measurements were also acquired along the length of one blade to identify the mode shapes of the turbine on that blade. Qualitatively reasonable results were obtained for seven modes of the turbine, which were found to correspond to a tower bending mode and the first two bending modes of the blades. It would have been preferable to have also measured the modes of the turbine by some other means so that the shapes obtained in that test could be validated, but that was not possible, so here they were only evaluated qualitatively.

It is informative to consider the effort and expense that would have been required to estimate the mode shapes of the turbine using conventional methods. In order to perform an OMA test using accelerometers, one would have to install sensors along the length of the blade and then run cables to a central data acquisition system (more than 30 meters if that was located on the ground). The sensors and cabling would mass load the blade to some extent, and all of the instrumentation would have to be removed after the test was complete. If conventional LDV were used to obtain the mode shapes, one would need at least one additional laser to serve as a reference. The other laser could then be roved to obtain the power spectrum at various points along the length of the blade. The laser must observe each point for at least 10 minutes to obtain a reasonable power spectrum, so a long test would be required to obtain even a few point measurements using that approach. Furthermore, the point measurements could be contaminated if the wind conditions and hence the excitation changed significantly as the test progressed from one point to the next. In contrast, the OMA-CSLDV method acquired reasonable mode shapes for several modes of the turbine from only two 10-minute time records (one fixed-point measurement at the tip and one $1.6 \mathrm{~Hz}$ CSLDV measurement).

\section{Acknowledgements}

This material is based on work supported by the National Science Foundation under Grant No. CMMI0969224. The authors also wish to thank Renewegy LLC, www.renewegy.com, for making their facilities available for the testing described here and for their help and support in this regard.

\section{References}

[1] M. S. Allen and D. M. Aguilar, "Model Validation of a Bolted Beam Using Spatially Detailed Mode Shapes Measured by Continuous-Scan Laser Doppler Vibrometry," in 50th AIAA/ASME/ASCE/AHS/ASC Structures, Structural Dynamics, and Materials Conference Palm Springs, California, 2009.

[2] $\quad$ P. Sriram, J. I. Craig, and S. Hanagud, "Scanning laser Doppler vibrometer for modal testing," International Journal of Analytical and Experimental Modal Analysis, vol. 5, pp. 155-167, 1990.

[3] P. Sriram, S. Hanagud, and J. I. Craig, "Mode shape measurement using a scanning laser doppler vibrometer." vol. 1 Florence, Italy: Publ by Union Coll, Schenectady, NY, USA, 1991, pp. 176-181.

[4] P. Sriram, S. Hanagud, and J. I. Craig, "Mode shape measurement using a scanning laser doppler vibrometer," International Journal of Analytical and Experimental Modal Analysis, vol. 7, pp. 169-178, 1992.

[5] C. W. Schwingshackl, A. B. Stanbridge, C. Zang, and D. J. Ewins, "Full-Field Vibration Measurement of Cylindrical Structures using a Continuous Scanning LDV Technique," in 25th International Modal Analysis Confernce (IMAC XXV) Orlando, Florida, 2007.

[6] A. B. Stanbridge and D. J. Ewins, "Modal testing using a scanning laser Doppler vibrometer," Mechanical Systems and Signal Processing, vol. 13, pp. 255-70, 1999.

[7] A. B. Stanbridge, M. Martarelli, and D. J. Ewins, "Measuring area vibration mode shapes with a continuous-scan LDV," Measurement, vol. 35, pp. 181-9, 2004.

[8] M. Martarelli, "Exploiting the Laser Scanning Facility for Vibration Measurements," Ph.D. Thesis, Imperial College of Science, Technology \& Medicine, London: Imperial College, 2001.

[9] A. B. Stanbridge, A. Z. Khan, and D. J. Ewins, "Modal testing using impact excitation and a scanning LDV," Shock and Vibration, vol. 7, pp. 91-100, 2000.

[10] S. Vanlanduit, P. Guillaume, and J. Schoukens, "Broadband vibration measurements using a continuously scanning laser vibrometer," Measurement Science \&amp; Technology, vol. 13, pp. 1574-82, 2002.

[11] M. S. Allen, "Frequency-Domain Identification of Linear Time-Periodic Systems using LTI Techniques," Journal of Computational and Nonlinear Dynamics, vol. 4, 24 Aug. 2009. 
[12] M. S. Allen and M. W. Sracic, "A New Method for Processing Impact Excited Continuous-Scan Laser Doppler Vibrometer Measurements," Mechanical Systems and Signal Processing, vol. 24, pp. 721-735, 2010.

[13] S. Yang, M. W. Sracic, and M. S. Allen, "Two algorithms for mass normalizing mode shapes from impact excited continuous-scan laser Doppler vibrometry," Journal of Vibration and Acoustics, submitted, 2010.

[14] M. S. Allen and M. W. Sracic, "Mass Normalized Mode Shapes Using Impact Excitation and ContinuousScan Laser Doppler Vibrometry," in 8th International Conference on Vibration Measurements by Laser Techniques Ancona, Italy, 2008, pp. 7098-3.

[15] M. S. Allen, M. W. Sracic, S. Chauhan, and M. H. Hansen, "Output-Only Modal Analysis of Linear Time Periodic Systems with Application to Wind Turbine Simulation Data," in 28th International Modal Analysis Conference (IMAC XXVIII) Jacksonville, Florida, 2010.

[16] M. S. Allen, M. W. Sracic, S. Chauhan, and M. H. Hansen, "Output-Only Modal Analysis of Linear Time Periodic Systems with Application to Wind Turbine Simulation Data," Mechanical Systems and Signal Processing, vol. submitted Oct. 2010, 2010.

[17] N. M. Wereley, "Analysis and Control of Linear Periodically Time Varying Systems," PhD Thesis, Department of Aeronautics and Astronautics, Cambridge: Massachusetts Institute of Technology, 1991.

[18] N. M. Wereley and S. R. Hall, "Linear time periodic systems: transfer functions, poles, transmission zeroes and directional properties," in Proceedings of the 1991 American Control Conference Boston, MA, USA: American Autom. Control Council, 1991, pp. 1179-84.

[19] N. M. Wereley and S. R. Hall, "Frequency response of linear time periodic systems," Honolulu, HI, USA, 1990, pp. 3650-3655.

[20] L. Hermans and H. Van Der Auweraer, "Modal Testing and Analysis of Structures Under Operational Conditions: Industrial Applications," Mechanical Systems and Signal Processing, vol. 13, pp. 193-216, 1999.

[21] E. Parloo, B. Cauberghe, F. Benedettini, R. Alaggio, and P. Guillaume, "Sensitivity-Based Operational Mode Shape Normalization: Application to A Bridge," in 22nd International Modal Analysis Conference (IMAC XXII) Dearborn, Michigan, 2004.

[22] B. Peeters and G. D. Roeck, "Stochastic System Identification for Operational Modal Analysis: A Review," Journal of Dynamic Systems, Measurement, and Control, vol. 123, December 2001.

[23] A. Fasana, L. Garibaldi, E. Giorcelli, and D. Sabia, "Z24 Bridge Dynamic Data Analysis by Time Domain Methods," in International Modal Analysis Conference (IMAC XIX) Kissimmee, Florida, 2001.

[24] A. Guyader and L. Mevel, "Covariance driven subspace methods : input/output vs output-only," in International Modal Analysis Conference (IMAC XXII) Dearborn, Michigan, 2004.

[25] M. Richardson and B. Schwarz, "Modal Parameter Estimation from Operating Data," in Sound and Vibration, 2003, pp. 28-36.

[26] G. H. James and T. G. Carne, "Damping measurements on operating wind turbines using the natural excitation technique (NExT)," in 11th ASME Wind Energy Symposium presented at the Energy Sources Technology Conference and Exhibition. vol. 12 Houston, TX, USA: Publ by ASME, New York, NY, USA, 1992, pp. 75-81.

[27] A. Gasparoni, M. S. Allen, S. Yang, M. W. Sracic, P. Castellini, and E. P. Tomasini, "Experimental Modal Analysis on a Rotating Fan Using Tracking-CSLDV," in 9th International Conference on Vibration Measurements by Laser and Noncontact Techniques Ancona, Italy, 2010.

[28] S. Yang, M. W. Sracic, and M. S. Allen, "Two algorithms for mass normalizing mode shapes from impact excited continuous-scan laser Doppler vibrometry," Journal of Vibration and Acoustics, vol. Submitted Aug 2010, 2010.

[29] S. Rothberg, "Numerical simulation of speckle noise in laser vibrometry," Applied Optics, vol. 45, pp. 4523-33, 2006.

[30] S. J. Rothberg, "Laser vibrometry. Pseudo-vibrations," Journal of Sound and Vibration, vol. 135, pp. 516522, 1989.

[31] M. S. Allen and J. H. Ginsberg, "A Global, Single-Input-Multi-Output (SIMO) Implementation of The Algorithm of Mode Isolation and Applications to Analytical and Experimental Data," Mechanical Systems and Signal Processing, vol. 20, pp. 1090-1111, 2006.

[32] S. Chauhan, M. H. Hansen, and D. Tcherniak, "Application of Operational Modal Analysis and Blind Source Separation / Independent Component Analysis Techniques to Wind Turbines," in 27th International Modal Analysis Conference (IMAC XXVII) Orlando, Florida: SPIE, 2009.

[33] J. Jonkman, "NREL 5 MW Baseline Wind Turbine," Technical report, NREL/NWTC, 1617 Cole Boulevard; Golden, CO 80401-3393, USA 2005. 